Marquette University

e-Publications@Marquette

Chemistry Faculty Research and Publications

Chemistry, Department of

$1-1-2014$

\title{
On Readout of Vibrational Qubits Using Quantum Beats
}

Dmytro Shyshlov

Marquette University

Eduardo Berrios

University of Illinois at Urbana-Champaign

Martin Gruebele

University of Illinois at Urbana-Champaign

Dmitri Babikov

Marquette University,dmitri.babikov@marquette.edu

Published version. Journal of Chemical Physics, Vol. 141, No. 22 (2014): 224306. DOI. (C) 2014

American Institute of Physics. Used with permission. 


\title{
On readout of vibrational qubits using quantum beats
}

\author{
Dmytro Shyshlov, ${ }^{1}$ Eduardo Berrios, ${ }^{2}$ Martin Gruebele, ${ }^{2}$ and Dmitri Babikov ${ }^{1, a)}$ \\ ${ }^{1}$ Chemistry Department, Marquette University, Milwaukee, Wisconsin 53201, USA \\ ${ }^{2}$ Department of Chemistry, Department of Physics and Center for Biophysics and Computational Biology, \\ University of Illinois, Urbana, Illinois 61801, USA
}

(Received 13 August 2014; accepted 19 November 2014; published online 9 December 2014)

\begin{abstract}
Readout of the final states of qubits is a crucial step towards implementing quantum computation in experiment. Although not scalable to large numbers of qubits per molecule, computational studies show that molecular vibrations could provide a significant (factor $2-5$ in the literature) increase in the number of qubits compared to two-level systems. In this theoretical work, we explore the process of readout from vibrational qubits in thiophosgene molecule, $\mathrm{SCCl}_{2}$, using quantum beat oscillations. The quantum beats are measured by first exciting the superposition of the qubit-encoding vibrational states to the electronically excited readout state with variable time-delay pulses. The resulting oscillation of population of the readout state is then detected as a function of time delay. In principle, fitting the quantum beat signal by an analytical expression should allow extracting the values of probability amplitudes and the relative phases of the vibrational qubit states. However, we found that if this procedure is implemented using the standard analytic expression for quantum beats, a non-negligible phase error is obtained. We discuss the origin and properties of this phase error, and propose a new analytical expression to correct the phase error. The corrected expression fits the quantum beat signal very accurately, which may permit reading out the final state of vibrational qubits in experiments by combining the analytic fitting expression with numerical modelling of the readout process. The new expression is also useful as a simple model for fitting any quantum beat experiments where more accurate phase information is desired. () 2014 AIP Publishing LLC. [http://dx.doi.org/10.1063/1.4903055]
\end{abstract}

\section{INTRODUCTION}

Quantum computation can be implemented by using shaped ultrafast laser pulses to control population transfer between molecular vibrational states. ${ }^{1-46}$ In one type of implementation, a set of $2^{n}$ vibrational eigenstates in a molecule can represent $n$ qubits that are inherently entangled, ${ }^{4}$ encoding several qubits on a single molecule, as shown in Fig. 1. High-fidelity quantum gates $2,3,9,16,17,40$ and simple quantum algorithms $\mathrm{s}^{8,13,20,30,41}$ have been investigated computationally for 2-5 vibrational qubits per molecule, scaling up by a useful amount the number of qubits per information carrier over atomic two-level systems. ${ }^{20}$

The result of a quantum computation leaves $n$ qubits encoded in the superposition of $2^{n}$ computational states. But how do we read out the state of vibrational qubits to complete the computation carried out in a molecule? The goal of the readout step is to obtain information about populations and phases of the computational states. It can be achieved by employing quantum beat spectroscopy as follows. The superposition of the computational qubit states is pumped to a single readout state by a time-delayed laser pulse. Fluorescence from the readout state is then detected. Repeating this for different time delays one obtains a periodic quantum beat signal (see insert in Fig. 1) from which populations and relative phases of the computational states can be extracted by fitting this signal with analytic expression.

\footnotetext{
a) Author to whom correspondence should be addressed. Electronic mail: Dmitri.Babikov@mu.edu.
}

This readout scheme is destructive, meaning that molecules in a beam, once excited to the readout state, lose their qubit information and cannot be excited again with a different time delay. We see two ways of circumventing this problem. One is to repeat preparation of the qubit system, which can be done reliably with modern pulsed lasers and beam-cooled molecules. The overhead (number of readouts) will depend on the total frequency bandwidth $\Delta E$ and on the smallest difference $\delta E$ among pairwise vibrational state energy differences roughly as $\Delta E / \delta E \cdot \ln (\Delta E / \delta E)$ if a fast Fourier transform algorithm is used to extract frequency components. This overhead is fixed and is not very large for $2-5$ vibrational qubits. Alternatively, it is possible to reduce the pulse power in order to excite only a small fraction of the molecules from the beam, which will permit reading out from the same ensemble of molecules several times, with different time delays. Moreover, other approaches to the readout of vibrational qubits, such as non-destructive wave packet interferometry, ${ }^{47}$ can also be considered in the future.

Another often discussed requirement to practical quantum computation is scaling - opportunity to increase the number of qubits to hundreds or even thousands. ${ }^{48,49}$ The prototypes of quantum computers created so far are still rather small. Latest achievements include 14 entangled qubits in the ion trap, ${ }^{50}$ five superconducting qubits, ${ }^{51}$ and only two atomic qubits in an optical lattice. ${ }^{52}$ Our goal with molecular qubits is not to replace methods such as atomic or ion trapping, but to enhance them by allowing 2-5 qubits per carrier instead of a single qubit. The possibility of encoding more than one 


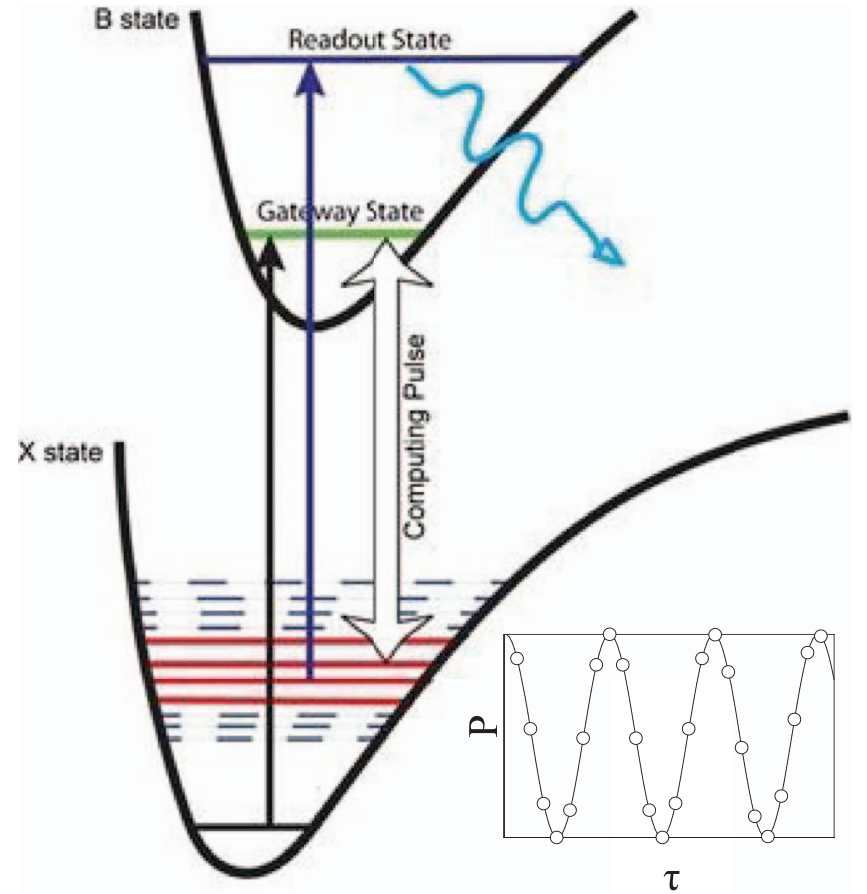

FIG. 1. The model of molecular qubits is thiophosgene $\mathrm{SCCl}_{2}$. Red lines show computational qubit states, dashed lines show vibrational eigenstates closest to the qubit states. Initialization is done by a laser pulse that excites molecule to the gateway state. The computational step is performed by a shaped laser pulse that circles population between the qubit states and an electronically excited gateway state until the final superposition is obtained. After computational step is finished the superposition of qubit states is excited to the readout state with time-delayed laser pulse. Fluorescence from the readout state is analyzed to extract information about populations and relative phases of qubit states. The inset demonstrates an example of quantum beat signal from one qubit, where circles indicate results of calculations and solid line is a numerical fit.

qubit has been demonstrated computationally for molecular ions. ${ }^{23}$ It seems quite feasible to create in the experiment a system of few vibrational qubits, fully entangled and well separated from the environment. ${ }^{4}$ Such prototype systems by themselves also could be used in the fundamental studies of quantum control, noise effects, decoherence, etc. For these small-scale (or test-bed) applications, the quantum beat approach to the readout, discussed in this paper, is also quite appropriate.

We consider the readout process using thiophosgene molecule, $\mathrm{SCCl}_{2}$ shown in Fig. 1, as our model for a molecular carrier of several qubits. This molecule has already been studied extensively as a platform for qubit manipulation, including such aspects as effects of rotation and laser power on population transfer ${ }^{20}$ optimal shape of high-fidelity computational pulses ${ }^{42}$ and their robustness, ${ }^{43}$ although readout has not been considered in detail before. Computational states (qubits, or q-words) are encoded in the vibrationally excited states in the ground electronic state of thiophosgene. The process of quantum computation itself is performed by a shaped laser pulse that cycles population between the computational states and the electronically excited "gateway" state (see Fig. 1). The shape of this computational pulse is optimized to implement a particular quantum gate, ${ }^{42}$ or an algorithm. ${ }^{20} \mathrm{An}-$ other electronically excited state is used as a readout state.
TABLE I. Energy spectrum and transition dipole moments of the computational states and the readout state in the model of thiophosgene molecule.

\begin{tabular}{lcccc}
\hline \hline State & $\begin{array}{r}\text { Energy } \\
\left(\mathrm{cm}^{-1}\right)\end{array}$ & $\begin{array}{c}\text { Transition } \\
\text { moment, } D\end{array}$ & $\begin{array}{c}\text { One-qubit } \\
\text { assignment }\end{array}$ & $\begin{array}{c}\text { Two-qubit } \\
\text { assignment }\end{array}$ \\
\hline Readout & 36025 & $\ldots$ & & \\
\multicolumn{2}{l}{ Computational states } & & & $|00\rangle$ \\
1 & 9197.0 & 0.174 & $|0\rangle$ & $|01\rangle$ \\
2 & 9230.7 & 0.176 & $|1\rangle$ & $|10\rangle$ \\
3 & 9247.1 & 0.185 & & $|11\rangle$ \\
4 & 9271.1 & 0.166 & & \\
\hline \hline
\end{tabular}

The difference in energy between the ground state, the readout state, and the computational states allows convenient UV/vis laser pulses to be used. Thus, there is no direct population transfer between the computational states, and only transitions from the computational states and to the readout state are significant.

As the simplest model system for the readout, we include two computational states in our calculations to study readout from one vibrational qubit, and four states for readout from the vibrational two-qubit system. The energy spectrum and transition dipole moments of our model are taken from the experiment ${ }^{53,54}$ and are given in Table I. The intensity of fluorescence from the readout state is linearly proportional to its population. Thus, we will analyze theoretically and computationally the population of the readout state after interaction with the time-delayed readout pulse.

The amplitudes and relative phases of computational states can be determined in principle by fitting the quantum beat signal with an analytical expression. In the simplest case of readout from one qubit, we would need to determine the amplitudes of two computational states $A_{1}$ and $A_{2}$ and a single phase difference $\Delta \varphi$. To do that we need to fit the quantum beat signal with the analytical expression, obtained based on time-dependent perturbation theory ${ }^{55}$

$$
\begin{aligned}
P(\tau)= & K \cdot\left(\varepsilon_{1}^{2} \mu_{1}{ }^{2} A_{1}{ }^{2}+\varepsilon_{2}{ }^{2} \mu_{2}{ }^{2} A_{2}{ }^{2}+2 \varepsilon_{1} \mu_{1} A_{1} \varepsilon_{2} \mu_{2} A_{2}\right. \\
& \times \cos (\Delta E \tau-\Delta \varphi))
\end{aligned}
$$

where $\varepsilon_{1}$ and $\varepsilon_{2}$ are laser pulse intensities at frequencies of transitions from two computational states to the readout state, $\mu_{1}$ and $\mu_{2}$ are corresponding transition dipole moments, $\Delta E$ $=E_{2}-E_{1}$ is the energy difference between the computational states in angular frequency units, $\tau$ is the variable time delay, and $K$ is a fitting coefficient. Similar ideas have been implemented in the past for readout of the electron-spin qubits in the solid-state environments, such as a doped diamond crystal or a semiconductor quantum dot. ${ }^{56,57}$

Variations of expression (1) are featured in a variety of publications on quantum beat spectroscopy. ${ }^{55,58-60}$ Applications of quantum beat spectroscopy to molecules are usually focused on determining the energy difference $\Delta E^{55,58,59}$ or on the extraction of population distribution, ${ }^{60}$ but rarely on the phase factor $\Delta \varphi \cdot{ }^{61}$ Our goal is to extract the values of amplitudes of the computational states, $A_{1}$ and $A_{2}$, and of the phase difference between them, $\Delta \varphi$ (see Eq. (1)). The phase is a 
critical piece of information about the qubit-encoding states, and thus the state of the qubits they represent.

The goal of this paper is twofold. One is to investigate the possibility of accurate determination of amplitudes and phase difference from Eq. (1) for quantum beat signals obtained from superpositions of vibrational states. Determining and, if possible, expanding the range of applicability of this simple formula also is important for many other applications of the quantum beat spectroscopy: ${ }^{55,58-60}$ while exact numerical simulations are quite possible on modern computers in many cases, simple analytical models can provide additional insight. The second goal is to test Eq. (1) computationally in the application to a qubit readout, using parameters of a suitable real molecule (thiophosgene) and realistic parameters of the readout pulse. In this paper, we use numerical simulation in order to mimic the process of experimental readout. Namely, we first produce the quantum beat signal numerically, using wavepacket propagation techniques, for some (known) final state of the vibrational qubit, or qubits. Then, we try to fit this signal with the expression (1) above in order to extract the values of $A_{1}, A_{2}$, and $\Delta \varphi$, now assumed to be unknown (the values of energies are assumed to be known, e.g., from another experiment). Repeating this procedure for various final qubit states, various pulse parameters, and even for various vibrational states used to represent qubits, we can determine the accuracy of this readout procedure. Our important finding is that using the known formula (1) for the fit does not allow determining the phase accurately. We analyze the origin of this phase error and propose a simple fix to the problem.

\section{NUMERICAL PROPAGATION}

To determine the result of interaction of the molecular system with a laser pulse, we propagate the Schrödinger equation

$$
i \hbar \frac{\partial}{\partial t} \Psi(t)=\left[\hat{H}_{0}-\mu \varepsilon(t)\right] \Psi(t),
$$

in the quantum representation for the molecule and the classical representation for the field. The initial condition of these equations is given by the state of the qubit we want to read out. To expand the time-dependent wave function, we use the vibrational eigenfunctions of the system $\psi_{n}(x)$ with the corresponding energy eigenvalues $E_{n}$ and time-dependent coefficients $c_{n}(t)$

$$
\Psi(x, t)=\sum_{n=1}^{N} c_{n}(t) \cdot \psi_{n}(x) \cdot e^{-i E_{n} t} .
$$

Substitution Eq. (3) into Eq. (2) leads to the equations for the coefficients $c_{n}(t)$

$$
\begin{aligned}
& \dot{c}_{n}^{R}(t)=\varepsilon(t) \sum_{m}\left(c_{m}^{R}(t) \sin \theta_{m, n}-c_{m}^{I}(t) \cos \theta_{m, n}\right) \cdot M_{m, n}, \\
& \dot{c}_{n}^{I}(t)=\varepsilon(t) \sum_{m}\left(c_{m}^{R}(t) \cos \theta_{m, n}-c_{m}^{I}(t) \sin \theta_{m, n}\right) \cdot M_{m, n},
\end{aligned}
$$

where $\theta_{m, n}=\left(E_{m}-E_{n}\right) t$ are phase shifts, $M_{m, n}=\left\langle\psi_{m}|\mu| \psi_{n}\right\rangle$ are the elements of the dipole moment matrix, and $c_{m}^{R}$ and $c_{m}^{I}$ are the real and imaginary parts of the complex coefficient $c_{m}$. At the end of the quantum computation, and just before interaction with the readout pulse, they represent the final state of vibrational qubit. $^{22}$

Population transfer from the qubit states to the readout state depends on the choice of the laser pulse $\varepsilon(t)$. We consider the readout pulse to be a transform-limited Gaussian pulse, created in the frequency domain

$$
\varepsilon(\omega)=\varepsilon_{\omega} \exp \left(-\frac{(|\omega|-\Omega)^{2}}{2 \sigma^{2}}\right),
$$

where $\Omega$ is the center frequency, $\varepsilon_{\omega}$ is the amplitude, and $\sigma$ is the width of the pulse in the frequency domain. The center frequency of the readout pulse $\Omega$ was set in the middle of two transition frequencies because for such a pulse the field intensities on both transition frequencies are equal. To obtain the time profile of the pulse $\varepsilon(t)$, we need to Fourier transform Eq. (5). Since the pulse must be purely real in the time domain, we need to prepare symmetrical pulse profile in the frequency domain. Therefore, we include negative frequencies in Eq. (5) and after Fourier transform we obtain a pulse that is real in the time domain

$$
\varepsilon(t)=\varepsilon_{\omega} \frac{\sigma}{\sqrt{2 \pi}} \exp \left(-\frac{\sigma^{2} t^{2}}{2}\right) \cos \Omega t .
$$

We can rewrite Eq. (6) for simplicity as

$$
\varepsilon(t)=\varepsilon_{0} f(t) \cos \Omega t,
$$

where we introduced

$$
\varepsilon_{0}=\varepsilon_{\omega} \frac{\sigma}{\sqrt{2 \pi}}
$$

and

$$
f(t)=\exp \left(-\frac{\sigma^{2} t^{2}}{2}\right) .
$$

Here, $\varepsilon_{0}$ is the pulse amplitude in the time domain, $\Omega$ is a carrier frequency, and $f(t)$ is the pulse envelope in the time domain, also a Gaussian.

The frequency resolution of the readout pulse is fixed at $2 \mathrm{~cm}^{-1}$ (a typical value for the experimental setup and ro-vibrational profiles of $\mathrm{SCCl}_{2}$ ). Frequency resolution determines pulse duration $T=16.68 \mathrm{ps}$, however duration of the highest intensity part of the pulse is significantly shorter ( $\sim 100 \mathrm{fs}$ ). The time grid for propagation was set in the range $(-T / 2, T / 2)$, while the pulse reaches its peak at $t=0$. The time delay between the shaped computation pulse (whose optimal shape was derived previously ${ }^{42,43}$ ) and the readout pulse is simply taken into account by the phases of the qubitencoding vibrational eigenstates (their phases evolve freely after the computation pulse is over while the population in each qubit encoding state remains constant).

Equations (4a) and (4b) are propagated using the RungeKutta method of 4 th order. To study the readout from one qubit, we included $N=3$ basis functions in Eq. (3), to account for two computational states and the readout state. This is the minimal number of states required for numerical test of 
Eq. (1). For readout from a two-qubit system, we included all five states given in Table I. In principle, one could study the influence of the interfering vibrational states of the molecule onto the readout process, by including more states into the basis set expansion, but this was not our goal here.

We tried two fitting methods. In one of them, we used four independent tuning parameters in Eq. (1): $K, A_{1}, A_{2}$, and $\Delta \varphi$, to fit the quantum beat signal one qubit, and normalized $A_{1}$ and $A_{2}$ after the fitting. In the second method, we had only three independent fitting parameters: $K, A_{1}$, and $\Delta \varphi$, while $A_{2}$ was computed from normalization as $A_{2}=\sqrt{1-A_{1}^{2}}$. We found that both methods produced identical results and, finally, adopted the second one, with smaller number of independent fitting parameters.

\section{RESULTS AND ANALYSIS}

For calculations with one qubit, the central frequency of the readout pulse was chosen at $\Omega=26811.15 \mathrm{~cm}^{-1}$, which is the middle between two frequencies for transitions from computational states 1 and 2 to the readout state (see Table I). Other parameters of the readout pulse were: the amplitude $\varepsilon_{\omega}=32$ a.u. and the width $\sigma=200 \mathrm{~cm}^{-1}$ in the frequency domain, which corresponds to the experimentally feasible pulse amplitude in the time domain $6 \times 10^{9} \mathrm{~V} / \mathrm{m}$, low enough to avoid ionization or excessive excited state absorption. The first set of numerical experiments was carried out for a model system (not a thiophosgene molecule), where we could vary energies of the computational states $E_{1}$ and $E_{2}$ in order to check how the energy difference $\Delta E$ affects the fitting of the quantum beat signal. Calculations with $\Delta E$ varying in the range from $15 \mathrm{~cm}^{-1}$ to $135 \mathrm{~cm}^{-1}$ are presented here. Note that for four states of thiophosgene in Table I the value of $\Delta E$ varies in the range from $16.4 \mathrm{~cm}^{-1}$ to $74.1 \mathrm{~cm}^{-1}$, with $\Delta E=33.7 \mathrm{~cm}^{-1}$ for states 1 and 2 chosen here to represent one qubit. So, the results of such model calculations are very relevant to the molecule we are studying. The energy of the readout state was fixed and equal to that of thiophosgene (see Table I). For simplicity, the state of the qubit was an equally weighted superposition of computational states, $A_{1}=A_{2}=\frac{1}{\sqrt{2}}$, with no phase difference, $\Delta \varphi=0$.

Once the simulation was run and the fit to Eq. (1) performed, we calculated the amplitude error as $\delta A=\frac{\left|A_{11}-A_{1}\right|}{1.00}$ $\times 100 \%$, where $A_{1}$ is the exact (known) amplitude, and $A_{1 f}$ is an amplitude obtained by fitting. The phase error was calculated as $\delta \varphi=\frac{\Delta \varphi_{f}-\Delta \varphi}{360^{\circ}} \times 100 \%$, where $\Delta \varphi$ is the exact (known) phase difference, and $\Delta \varphi_{f}$ is a phase difference obtained by fitting. Our results are presented in Figs. 2 and 3. We found that the amplitude error $\delta A$ of the fitting increases roughly exponentially with energy difference $\Delta E$ (see Fig. 2), but the value of $\delta A$ remains relatively small even for large $\Delta E$ and does not exceed $10^{-2} \%$ in our tests. For energy difference between computational states 1 and 2 in thiophosgene, $\Delta E=33.7 \mathrm{~cm}^{-1}$ (see Table I), the amplitude error would be $\sim 5 \times 10^{-3} \%$. Thus, we can confidently conclude that it should be possible to determine the values of amplitudes of the qubit states with high precision.

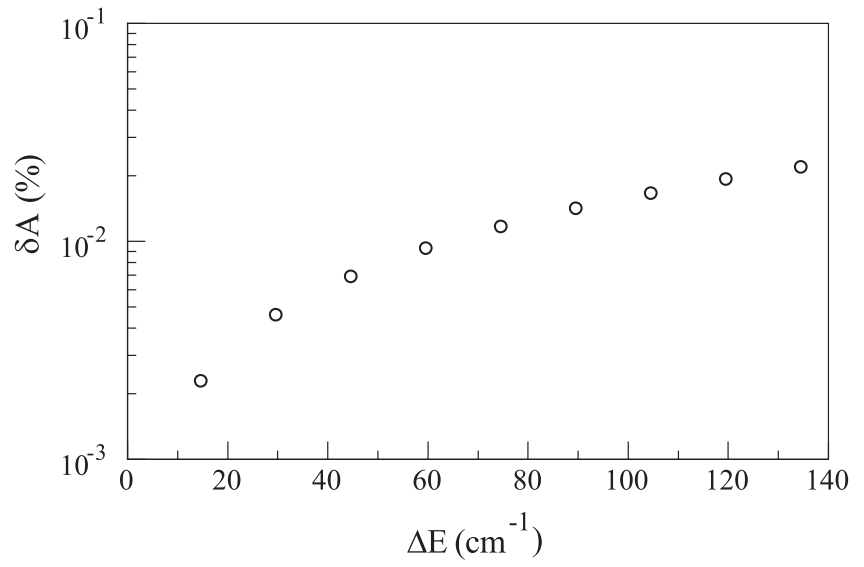

FIG. 2. Amplitude error as a function of energy difference between computational states in a model system. Pulse amplitude is at $\varepsilon_{\omega}=32$ a.u. Pulse width is $\sigma=200 \mathrm{~cm}^{-1}$.

However, we found that the value of phase error $\delta \varphi$ is significantly higher than that of the amplitude error. Figure 3 shows that $\delta \varphi$ increases roughly linearly with energy difference $\Delta E$ and reaches almost $5 \%$ at $\Delta E=135 \mathrm{~cm}^{-1}$. For thiophosgene with $\Delta E=33.7 \mathrm{~cm}^{-1}$, the phase error would be $\sim 1.2 \%$. We also performed a series of fitting tests for nonzero values of the phase difference $\Delta \varphi$ between the qubit states, increasing it gradually up to $180^{\circ}$ (all with $A_{1}=A_{2}$ $=\frac{1}{\sqrt{2}}$ ). In all those tests, we found that phase error $\delta \varphi$ does not depend on the value of phase difference $\Delta \varphi$ itself, but depends on energy difference $\Delta E$, and the dependence $\delta \varphi(\Delta E)$ is nearly identical to that in Fig. 3 (i.e., close to linear).

In order to check whether this non-negligible phase error $\delta \varphi$ depends on pulse parameters, we then performed a series of fitting tests for a system with constant energy difference $\Delta E=30 \mathrm{~cm}^{-1}$ by varying parameters of the readout pulse, such as width in the frequency domain $\sigma$ and amplitude $\varepsilon_{\omega}$. The quantum beat signals obtained in those calculations again were fitted using expression (1). The dependence of phase error on the pulse width $\sigma$ is shown in Fig. 4(a) (for pulse amplitude fixed at $\varepsilon_{\omega}=32$ a.u.) and one can see that pulses with larger $\sigma$ give lower phase error in the fitting.

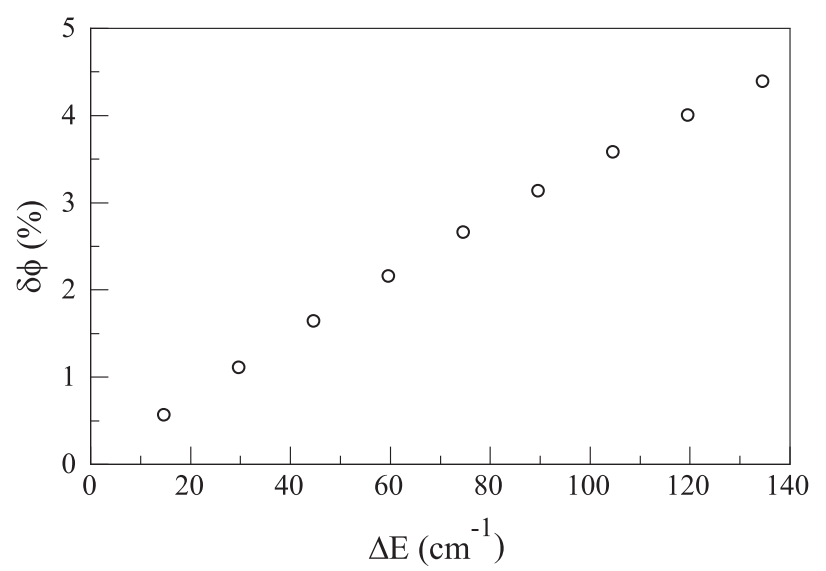

FIG. 3. Phase error as a function of energy difference between computational states in a model system. Pulse amplitude is at $\varepsilon_{\omega}=32$ a.u. Pulse width is $\sigma=200 \mathrm{~cm}^{-1}$. 

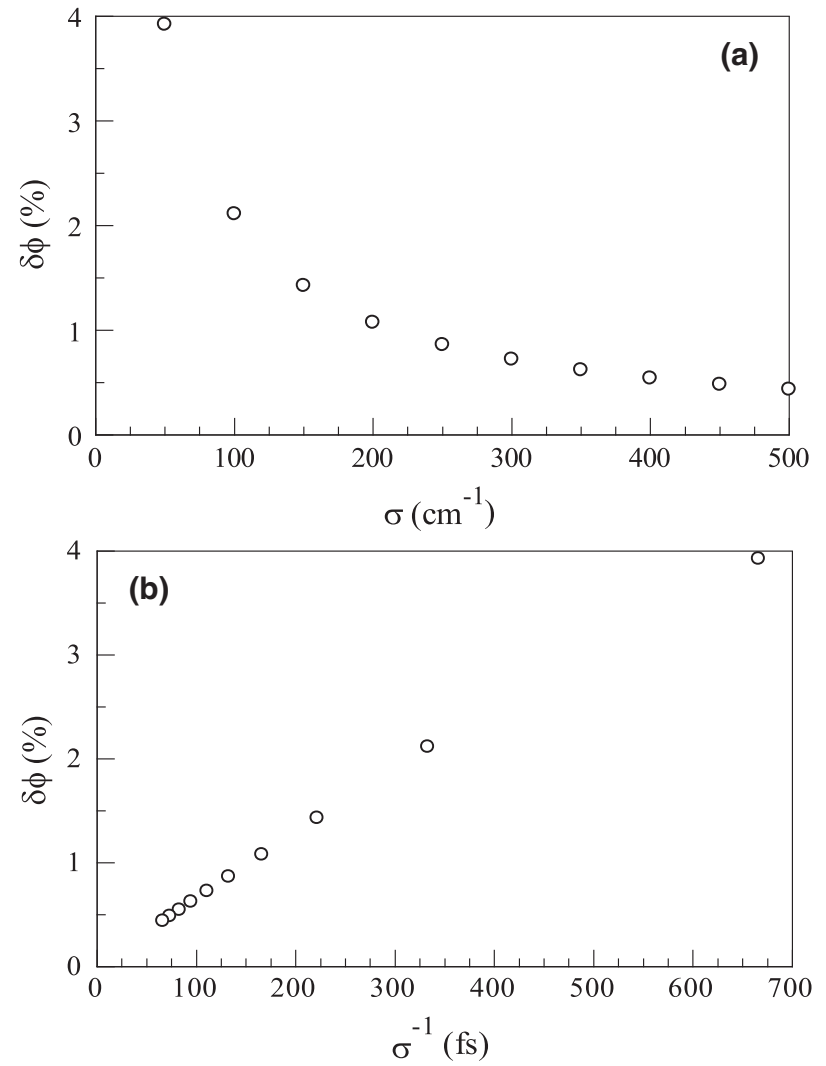

FIG. 4. Phase errors obtained for different values of the width of the readout pulse (in the frequency domain). Frame (a) shows dependence of phase error on pulse width, while frame (b) shows dependence on the inversed width as a parameter of pulse duration (in time domain). Energy difference between the computational states is $\Delta E=30 \mathrm{~cm}^{-1}$. Pulse amplitude is fixed at $\varepsilon_{\omega}=32$ a.u.

According to Eqs. (5) and (6), the pulse width in the frequency domain $\sigma$ corresponds to the pulse width in the time domain $1 / \sigma$. Therefore, the value of inversed pulse width $1 / \sigma$ is a parameter that describes duration of the pulse. The dependence of phase error $\delta \varphi$ on the inverse pulse width $1 / \sigma$ is shown on Fig. 4(b). The dependence is nearly linear and we can conclude that longer readout pulses with a narrower frequency profile result in a larger phase error.

In order to study dependence of the phase error $\delta \varphi$ on the pulse amplitude $\varepsilon_{\omega}$, we fixed the pulse width at $\sigma=200 \mathrm{~cm}^{-1}$, and varied the value of pulse amplitude $\varepsilon_{\omega}$ in Eq. (8) through the range shown in Fig. 5. For each case, we carried out an independent calculation and determined the value of $\delta \varphi$. We found that pulses with larger amplitudes results in larger phase errors and, through the studied range of values, the dependence is close to exponential. The phase error is on the order of $\delta \varphi \approx 2 \%$ for $\varepsilon_{\omega}<40$ a.u., but it drastically increases for larger values of pulse amplitudes, and, reaches $\delta \varphi \approx 50 \%$ for $\varepsilon_{\omega}=65$ a.u. This illustrates that the phase error we observed and studied may be significant even for pulse energies relevant to experiment.

We conclude from these results that the origin of the phase error is in the fact that the readout pulse has nonzero duration and fluence, violating the assumptions of timedependent perturbation theory used in Ref. 55. According to Eq. (3) a phase shift should occur between states 1 and 2, and

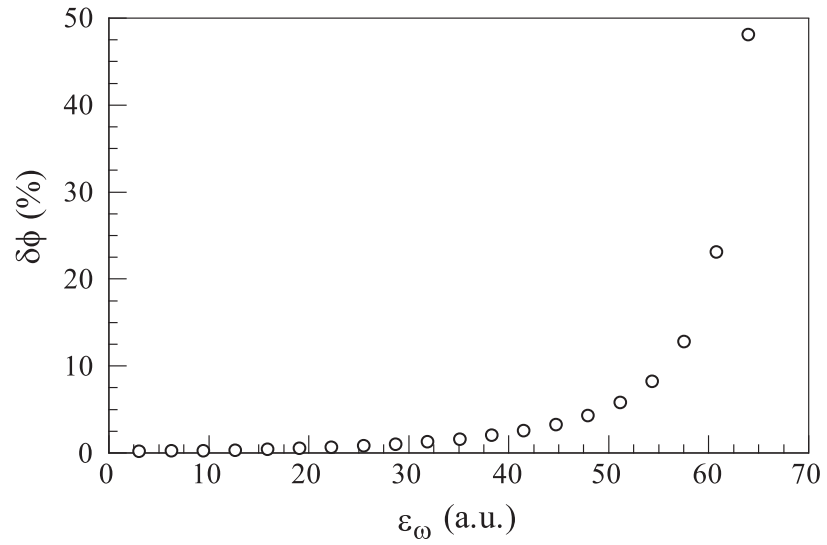

FIG. 5. Phase errors obtained for different values of the amplitude of the readout pulse (in the frequency domain). Energy difference between the computational states is $\Delta E=30 \mathrm{~cm}^{-1}$. Pulse width is fixed at $\sigma=200 \mathrm{~cm}^{-1}$.

it would be more pronounced for longer pulses and larger energy differences $\Delta E$, consistent with our observations. Therefore, the quantum beat signal obtained with a readout pulse of finite duration (rather than sudden pulse) in a system with non-degenerate levels $(\Delta E \neq 0)$ includes an additional phase shift. There is no such a phase shift in Eq. (1), which results in a phase error during fitting. In order to decrease this error one may want to use shorter readout pulses, which corresponds to increasing pulse width $\sigma$ in the frequency domain, as well as using pulses with lower amplitude or choosing states with smaller energy difference $\Delta E$ to represent qubits. However, the ideal case of an arbitrarily low pulse power and an arbitrarily short pulse duration cannot be achieved in real experiments. Typical to our experimental setup, and to gas-phase molecule fluorescence detection in general, are 10-100 $\mu \mathrm{J}$ pulses of duration $\sim 100 \mathrm{fs}$ focused to a Gaussian waist of $w \sim 10 \mu \mathrm{m}$. The peak electric field they produce is $E_{\text {peak }}$ $\sim 10^{10} \mathrm{~V} / \mathrm{m}$, which corresponds to $\varepsilon_{\omega} \sim 150-450$ a.u. in Eqs. (5) and (6). From Fig. 5 we see that at such conditions the phase error is significant, unfortunately.

On a practical note, the readout scheme considered here has the advantage that background effects in the readout can be eliminated. The readout is a single photon, relatively lowfield measurement, whose lowest power is determined by the signal-to-noise ratio required to detect fluorescence and fit the quantum beats. Our measurements of jet-cooled thiophosgene $\tilde{X} \rightarrow \tilde{B}$ excitation in a time-of-flight mass spectrometer show negligible ionization for $285 \mathrm{~nm}$ (tripled output of a regenerative amplifier) as well as for the visible pulses $\sim 400 \mathrm{~nm}$ that have been proposed for readout of excited vibrational states in the $\tilde{X}$ electronic state to the $\tilde{B}$ electronic state. ${ }^{20}$ The main practical problem for both ns and fs excitation pulses is the excited state absorption from the readout state in the $\tilde{B}$ electronic state. This reduces the population in the readout state that can be monitored by fluorescence or stimulated emission pumping. ${ }^{62}$ It does not produce a fluorescence signal that interferes with readout because the highly excited states decay non-radiatively. For this reason, the calculations proposed here correspond to maximum $E$-fields in the time domain of $10^{9}-10^{10} \mathrm{~V} / \mathrm{m}$, close to experimental fields that produce strong fluorescence signals but not excessive 
ionization or excited state absorption. As discussed above, the low peak intensities are advantageous because they minimize the exponential growth of phase error with probe-pulse energy, but in practice they need to be high enough to allow fluorescence detection of quantum beats.

So, instead of reducing the pulse power, we propose to compensate for phase error by calculating it numerically for a given system $(\Delta E)$ and pulse parameters $\left(\varepsilon_{\omega}, \sigma\right)$ and introducing it explicitly into the fitting expression (1) as a constant phase correction term $\varphi_{\text {corr }}$

$$
\begin{aligned}
P(\tau)= & K \cdot\left(\varepsilon_{1}^{2} \mu_{1}^{2} A_{1}^{2}+\varepsilon_{2}^{2} \mu_{2}^{2} A_{2}^{2}+2 \varepsilon_{1} \mu_{1} A_{1} \varepsilon_{2} \mu_{2} A_{2}\right. \\
& \left.\times \cos \left(\Delta E \tau-\Delta \varphi+\varphi_{\text {corr }}\right)\right)
\end{aligned}
$$

Using the fitting expression (10) with phase correction $\varphi_{\text {corr }}$ allows us to fit the phase $\Delta \varphi$ between the computational states with high precision. In order to do that we would need, first, to fit one quantum beats signal produced numerically for one arbitrary set of initial conditions $\left(A_{1}, A_{2}\right.$, and $\Delta \varphi$ ) using expression (1) in order to determine the phase error $\varphi_{\text {corr }}$ specific to energy difference of computational states $\Delta E$ and parameters of the readout pulse $\varepsilon_{\omega}$ and $\sigma$. This phase error does not depend on state populations $\left(A_{1}, A_{2}\right)$ or on their phase difference $\Delta \varphi$ and can be used in expression (10) to fit all the quantum beats signals produced from the given system with the given readout pulse. In particular, this procedure can be used to analyze the experimental quantum beats signal to derive $A_{1}, A_{2}$, and $\Delta \varphi$ in the experimentally implemented quantum computation, based on theoretically (numerically) derived value of $\varphi_{\text {corr }}$.

We tested the fitting approach proposed above using states 1 and 2 of thiophosgene, $\Delta E=33.7 \mathrm{~cm}^{-1}$ (see Table I) and the pulse with $\Omega=26811.15 \mathrm{~cm}^{-1}, \varepsilon_{\omega}=32$ a.u., and $\sigma=200 \mathrm{~cm}^{-1}$. First, using the amplitudes $A_{1}=A_{2}=\frac{1}{\sqrt{2}}$ and the phase difference $\Delta \varphi=0$ we produced one quantum beat signal and fitted it by Eq. (1) to determine the value of $\varphi_{\text {corr }}=4.42^{\circ}$. Next, we produced a number of different quantum beat signals for the same qubit and pulse, but with various populations of qubit states and various phase differences. Many combinations of $A_{1}=\sqrt{0.9}, \sqrt{0.8}, \sqrt{0.7}, \ldots, \sqrt{0.1}$ (and $A_{2}=\sqrt{0.1}, \sqrt{0.2}, \sqrt{0.3}, \ldots, \sqrt{0.9}$, respectively) with phase differences $\Delta \varphi=0^{\circ}, 22.5^{\circ}, 45^{\circ}, \ldots, 180^{\circ}$ were tried, which covers the surface of Bloch sphere with 81 sample points (by a $9 \times 9$ grid). All these quantum beat signals were fitted using the expression (10) with $\varphi_{\text {corr }}=4.42^{\circ}$. These tests confirmed that using the fitting expression (10), with properly determined phase correction, reduces phase error below $10^{-9} \%$ for any arbitrary state of vibrational qubit. Thus, our correction method is universally applicable to any output of the quantum computation procedure.

In order to test that this correction procedure will be valid for the pulses of higher intensities too, we have repeated all the tests described in the previous paragraph with several higher intensity readout pulses, up to the ionization limit estimated as $E_{\text {peak }} \sim 2.3 \times 10^{11} \mathrm{~V} / \mathrm{m}$, or $\varepsilon_{\omega}=1226$ a.u. We found that the phase correction $\varphi_{\text {corr }}$, determined for a given pulse and one arbitrarily chosen state of the qubit, remains universal and works for any other state of the qubit, as long as the same readout pulse is used, up to ionization limit of thiophosgene.

The same approach of fitting the quantum beat signal with phase correction can be used in order to readout a state of a two-qubit system, implemented using four vibrational states. To perform readout from four vibrational states, one would need to determine four amplitudes: $A_{1}, A_{2}, A_{3}$, and $A_{4}$, and six phase differences between each pair of computational states: $\Delta \varphi_{12}, \Delta \varphi_{13}, \Delta \varphi_{14}, \Delta \varphi_{23}, \Delta \varphi_{24}$, and $\Delta \varphi_{34}$. The fitting expression for two-qubit system is

$$
\begin{aligned}
P(\tau)= & K \cdot\left(\sum_{i=1,4} \varepsilon_{i}^{2} \mu_{i}^{2} A_{i}^{2}\right. \\
& +2 \sum_{i=1,3} \sum_{j=i+1,4} \varepsilon_{i} \mu_{i} A_{i} \varepsilon_{j} \mu_{j} A_{j} \\
& \left.\times \cos \left(\Delta E_{i j} \tau-\Delta \varphi_{i j}+\varphi_{\text {corr }_{i j}}\right)\right),
\end{aligned}
$$

where we introduced four phase corrections. Energies and transition dipole moments of vibrational states of thiophosgene we used in our calculations are given in Table I. Parameters of the readout pulse were: $\Omega=26788.53 \mathrm{~cm}^{-1}$ (average of four transition frequencies), $\varepsilon_{\omega}=32$ a.u., and $\sigma=200 \mathrm{~cm}^{-1}$. First, we calculated the quantum beat signal for equally weighted superposition $A_{1}=A_{2}=A_{3}=A_{4}=$ $\frac{1}{\sqrt{2}}$, with no phase differences $\Delta \varphi_{12}=\Delta \varphi_{13}=\Delta \varphi_{14}=\Delta \varphi_{23}$ $=\Delta \varphi_{24}=\Delta \varphi_{34}=0$. This signal was fitted using Eq. (11) in order to find the values of phase corrections for each oscillating term: $\varphi_{\text {corr } 12}=12.59^{\circ}, \varphi_{\operatorname{corr} 13}=18.87^{\circ}, \varphi_{\text {corr } 14}=$ $27.83^{\circ}, \varphi_{\text {corr } 23}=6.28^{\circ}, \varphi_{\text {corr } 24}=15.23^{\circ}, \varphi_{\text {corr } 34}=8.95^{\circ}$. Fitting with phase correction was then applied to a number of quantum beat signals, generated from an arbitrary superposition of computational states with different amplitudes and phase differences. Values of the phase error determined by fitting with Eq. (11) and numerically determined phase corrections were always below $10^{-9} \%$. These results confirm that accurate fitting of relative phases of computational states is possible in the case of the readout from a system of more than one vibrational qubit.

\section{CONCLUSIONS}

In this theoretical/computational work, we explored the process of readout from vibrational qubits in thiophosgene molecule, $\mathrm{SCCl}_{2}$, using quantum beat oscillations. It is assumed that the quantum beats are obtained by excitation of a superposition of the qubit states with time delayed pulses to the electronically excited readout state, followed by detecting oscillations of population of the readout state, through fluorescence, as a function of time delay of the readout pulse. We found that a significant phase error is obtained if the known analytic expression for quantum beats from time-dependent density matrix perturbation theory, Ref. 55, is used for extracting the values of probability amplitudes and relative phases of the vibrational qubit states. We discussed the source and the properties of this phase error and proposed an improved analytical expression, where a universal phase correction is 
introduced. Application of this new expression fixes the phase problem and allows fitting the quantum beat signal very accurately. It should permit to readout the final states of vibrational qubits in the experiments using an approach in which the analytic expression for fitting is combined with numerical modelling of the readout process, for computing the phase correction. Thus, real laser pulses of finite duration and fluence can, in principle, be used to readout vibrational qubits.

\section{ACKNOWLEDGMENTS}

This work was supported by the National Science Foundation (NSF), Grant No. CHE-1012075. This research used resources of the National Energy Research Scientific Computing Center, which is supported by the Office of Science of the (U.S.) Department of Energy (DOE) under Contract No. DE-AC02-05CH11231. Dmytro Shyshlov was supported in part by Eisch fellowship, and gratefully acknowledges the generosity of Dr. John J. Eisch.

${ }^{1}$ C. M. Tesch, L. Kurtz, and R. de Vivie-Riedle, Chem. Phys. Lett. 343, 633 (2001).

${ }^{2}$ C. M. Tesch and R. de Vivie-Riedle, Phys. Rev. Lett. 89, 157901 (2002).

${ }^{3}$ Z. Amitay, R. Kosloff, and S. R. Leone, Chem. Phys. Lett. 359, 8 (2002).

${ }^{4}$ J. Vala, Z. Amitay, B. Zhang, S. R. Leone, and R. Kosloff, Phys. Rev. A 66, 062316 (2002).

${ }^{5}$ U. Troppmann, C. M. Tesch, and R. de Vivie-Riedle, Chem. Phys. Lett. 378, 273 (2003).

${ }^{6}$ J. P. Palao and R. Kosloff, Phys. Rev. Lett. 89, 188301 (2002).

${ }^{7}$ J. P. Palao and R. Kosloff, Phys. Rev. A 68, 062308 (2003).

${ }^{8}$ C. M. Tesch and R. de Vivie-Riedle, J. Chem. Phys. 121, 12158 (2004).

${ }^{9}$ D. Babikov, J. Chem. Phys. 121, 7577 (2004).

${ }^{10}$ B. Korff, U. Troppmann, K. Kompa, and R. de Vivie-Riedle, J. Chem. Phys. 123, 244509 (2005).

${ }^{11}$ U. Troppmann and R. de Vivie-Riedle, J. Chem. Phys. 122, 154105 (2005).

${ }^{12}$ Y. Ohtsuki, Chem. Phys. Lett. 404, 126 (2005).

${ }^{13}$ Y. Ohtsuki, New J. Phys. 12, 045002 (2010).

${ }^{14}$ S. Suzuki, K. Mishima, and K. Yamashita, Chem. Phys. Lett. 410, 358 (2005).

${ }^{15}$ U. Troppmann, C. Gollub, and R. de Vivie-Riedle, New J. Phys. 8, 100 (2006).

${ }^{16}$ T. Cheng and A. Brown, J. Chem. Phys. 124, 034111 (2006).

${ }^{17}$ Y. Teranishi, Y. Ohtsuki, K. Hosaka, H. Chiba, H. Katsuki, and K. Ohmori, J. Chem. Phys. 124, 114110 (2006).

${ }^{18}$ K. Shioya, K. Mishima, and K. Yamashita, Mol. Phys. 105, 1283 (2007).

${ }^{19}$ M. Ndong, D. Lauvergnat, X. Chapuisat, and M. Desouter-Lecomte, J. Chem. Phys. 126, 244505 (2007).

${ }^{20}$ D. Weidinger and M. Gruebele, Mol. Phys. 105, 1999 (2007).

${ }^{21}$ C. Menzel-Jones and M. Shapiro, Phys. Rev. A 75, 052308 (2007).
${ }^{22}$ M. Zhao and D. Babikov, J. Chem. Phys. 126, 204102 (2007).

${ }^{23}$ D. Weidinger and M. Gruebele, Chem. Phys. 350, 139 (2008).

${ }^{24}$ L. Bomble, D. Lauvergnat, F. Remacle, and M. Desouter-Lecomte, J. Chem. Phys. 128, 064110 (2008).

${ }^{25} \mathrm{M}$. Tsubouchi and T. Momose, Phys. Rev. A 77, 052326 (2008).

${ }^{26}$ Y. Y. Gu and D. Babikov, J. Chem. Phys. 131, 034306 (2009).

${ }^{27}$ M. Schröder and A. Brown, J. Chem. Phys. 131, 034101 (2009).

${ }^{28}$ K. Mishima and K. Yamashita, Chem. Phys. 361, 106 (2009).

${ }^{29}$ K. Mishima and K. Yamashita, J. Chem. Phys. 130, 034108 (2009).

${ }^{30}$ K. Mishima and K. Yamashita, Chem. Phys. 367, 63 (2010).

${ }^{31}$ Q. Wei, S. Kais, and Y. P. Chen, J. Chem. Phys. 132, 121104 (2010).

${ }^{32}$ R. R. Zaari and A. Brown, J. Chem. Phys. 132, 014307 (2010).

${ }^{33}$ K. Mishima and K. Yamashita, Chem. Phys. 379, 13 (2011).

${ }^{34}$ R. R. Zaari and A. Brown, J. Chem. Phys. 135, 044317 (2011).

${ }^{35}$ Q. Wei, S. Kais, B. Friedrich, and D. Herschbach, J. Chem. Phys. 134, 124107 (2011)

${ }^{36}$ Q. Wei, S. Kais, B. Friedrich, and D. Herschbach, J. Chem. Phys. 135, 154102 (2011)

${ }^{37}$ P. Pellegrini, S. Vranckx, and M. Desouter-Lecomte, Phys. Chem. Chem. Phys. 13, 18864 (2011).

${ }^{38}$ P. Pellegrini and M. Desouter-Lecomte, Eur. Phys. J. D 64, 163 (2011).

${ }^{39}$ L. Wang and D. Babikov, Phys. Rev. A 83, 022305 (2011).

${ }^{40}$ L. Wang and D. Babikov, Phys. Rev. A 83, 052319 (2011).

${ }^{41}$ L. Wang and D. Babikov, J. Chem. Phys. 137, 064301 (2012).

${ }^{42}$ E. Berrios, M. Gruebele, D. Shyshlov, L. Wang, and D. Babikov, J. Phys. Chem. A 116(46), 11347 (2012).

${ }^{43}$ D. Shyshlov and D. Babikov, J. Chem. Phys. 137, 194318 (2012).

${ }^{44}$ R. R. Zaari and A. Brown, J. Chem. Phys. 137, 104306 (2012).

${ }^{45}$ J. Zhu, S. Kais, Q. Wei, D. Herschbach, and B. Friedrich, J. Chem. Phys. 138, 024104 (2013).

${ }^{46}$ A. Jaouadi, E. Barrez, Y. Justum, and M. Desouter-Lecomte, J. Chem. Phys. 139, 014310 (2013).

${ }^{47}$ M. B. Campbell, T. J. Bensky, and R. R. Jones, Opt. Express 1, 197 (1997).

${ }^{48}$ D. Kielpinski, C. Monroe, and D. J. Wineland, Nature (London) 417, 709 (2002).

${ }^{49}$ M. Saffman and K. Molmer, Phys. Rev. A 78, 012336 (2008).

${ }^{50}$ T. Monz et al., Phys. Rev. Lett. 106, 130506 (2011).

${ }^{51}$ R. Barends et al., Nature (London) 508, 500 (2014).

${ }^{52}$ M. Anderlini, P. J. Lee, B. L. Brown, J. Sebby-Strabley, W. D. Phillips, and J. V. Porto, Nature (London) 448, 452 (2007).

${ }^{53}$ E. Berrios and M. Gruebele, J. Phys. Chem. A 117, 7535 (2013).

${ }^{54}$ E. Berrios, S. Pratt, P. Tripathi, and M. Gruebele, J. Phys. Chem. A 117, 12082 (2013).

${ }^{55}$ M. Gruebele and A. H. Zewail, J. Chem. Phys. 98, 883 (1993).

${ }^{56}$ L. Jiang et al., Science 326, 267 (2009).

${ }^{57}$ K. Muller et al., Sci. Rep. 3, 1906 (2013).

${ }^{58}$ E. Hack and J. R. Huber, Int. Rev. Phys. Chem. 10, 287 (1991).

${ }^{59}$ M. Hayashi et al., Tamkang J. Sci. Eng. 3, 145 (2000).

${ }^{60}$ H. Goto, H. Katsuki, H. Ibrahim, H. Chiba, and K. Ohmori, Nat. Phys. 7, 383 (2011).

${ }^{61}$ H. Katsuki, K. Hosaka, H. Chiba, and K. Ohmori, Phys. Rev. A 76, 013403 (2007).

${ }^{62}$ B. Strickler and M. Gruebele, Phys. Chem. Chem. Phys. 6, 3786 (2004). 